\title{
An infant with orange-colored urine
}

\author{
Serwet Demirdas • Cornelis H. Schröder
}

Received: 15 September 2009 /Revised: 21 September 2009/Accepted: 21 September 2009/Published online: 21 October 2009

(C) IPNA 2009

Sirs,

Changes of the color of urine frequently occur. Most common is the white purulent appearance in urinary tract infections but also a red color due to hematuria or-mainly in infants-uric acid. Mere orange discoloration is, however, very rare.

An 11-month-old male infant presented with orangestained diapers. He was otherwise in good health, and the parents reported no additional complaints. History revealed that the symptoms started several months before presentation. At first onset of complaints, the staining occurred once or twice a week. During the 3 weeks prior to presentation at our clinic, the frequency increased to a daily basis; generally occurring in the morning. Physical examination was unremarkable. Urine showed no erythrocytes, leukocytes, or protein in the routine examination. Uric acid was $1.2 \mathrm{mmol} / \mathrm{l}$ and therefore within the normal range for the patient's age. Urine culture revealed a significant amount $\left[>10^{5}\right.$ colony-forming units $\left.(\mathrm{CFU}) / \mathrm{ml}\right]$ of Citrobacter sedlakii.

In the literature, there are no known case reports describing orange-coloured urine due to bacterial overgrowth in the bladder. C. sedlakii is known to inhabit human and animal intestines, water, soil, sewage, and sometimes food [1]. The genus Citrobacter has been known

S. Demirdas $(\varangle) \cdot$ C. H. Schröder

Gelre ziekenhuizen, General Pediatrics,

Albert Schweitzerlaan 31,

Apeldoorn, The Netherlands

e-mail: s.demirdas@gelre.nl to cause infection in the neonate and immunocompromised patients. More serious infections in the neonate, such as meningitis, sepsis, and cerebral abscesses, have been described [2]. C. sedlakii is part of the tribe Citrobactereae. These are gram-negative bacilli of the family Enterobacteriaceae. C. sedlakii produces indole [3] and this characteristic is used in laboratories to distinguish the species from other organisms within the Citrobactereae tribe [4]. Indole is excreted by the bacteria as a result of degradation of tryptophan and is characterized by its orange color. In our infant, this orange excretion caused stained diapers, mainly in the morning, as a result of the possibility for the bacteria to grow within the bladder over night. Due to the bacterial overgrowth, the patient was treated with cotrimoxazole for 7 days. Treatment resulted in symptom disappearance.

Our case suggests that orange-stained urine in infants may sporadically be related to bacterial overgrowth by $C$. sedlakii. The characteristic of indole production explains how the urine of our patient could have been colored when the urinary tract was colonized by $C$. sedlakii. It is the first time that such a discoloration of urine has been described.

\section{References}

1. Doran TI (1999) The role of Citrobacter in clinical disease of children: review. Clin Infect Dis 28:384-394

2. Dyer J, Hayani KC, Janda WM, Schreckenberger PC (1997) Citrobacter sedlakii meningitis and brain abscess in a premature infant. J Clin Microbiol 35:2686-2688

3. Borenshtein D, Schauer DB (2006) The genus Citrobacter. Prokaryotes 6:90-98

4. Janda JM, Abbott SL, Cheung WKW, Hanson DF (1994) Biochemical identification of Citrobacteria in clinical laboratory. J Clin Microbiol 32:1850-1854 